\title{
The role of per cent predicted 6-min walk distance in pulmonary arterial hypertension
}

\author{
W-T.N. Lee, A.J. Peacock and M.K. Johnson
}

ABSTRACT: Absolute 6-min walk distance (6MWD) predicts mortality in pulmonary arterial hypertension (PAH), but varies greatly between normal individuals due to physiological factors such as age, sex, height and weight. The \% predicted 6MWD adjusts for these factors and may predict mortality more reliably. The aim of the study was to compare the strength of mortality prediction by absolute and \% predicted 6MWD in PAH at baseline and on treatment.

$\%$ predicted 6MWD was calculated using four different reference equations in 137 PAH patients (idiopathic and connective tissue disease associated) diagnosed between November 2000 and November 2009. Cox proportional hazards and receiver-operating characteristic (ROC) analyses were used to compare the prognostic strength of absolute and \% predicted 6MWD.

$\%$ predicted 6MWD was predictive of all-cause mortality at baseline (hazard ratio $0.74-0.83$ per $10 \%$ increase; $p<0.05)$ and on treatment $(0.67-0.75$ per $10 \%$ increase; $p<0.01)$, but each respective area under the ROC curve was not different from that of absolute 6MWD for predicting 2-yr mortality at baseline (absolute versus \% predicted: 0.74 versus $\mathbf{0 . 7 1 - 0 . 7 5 )}$ ) or on treatment (0.77 versus $0.72-0.78)$.

In conclusion, \% predicted 6MWD may help clinicians interpret the 6-min walk test, but its prognostic value is not superior to that of absolute 6MWD.

KEYWORDS: 6-min walk distance, mortality, pulmonary hypertension

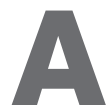

bsolute 6-min walk distance (6MWD) has been established as the principal clinical outcome measure in pulmonary arterial hypertension $(\mathrm{PAH})$ and has been used as the primary end-point in most clinical trials of new PAH therapies [1]. As a measure of submaximal exercise capacity, it correlates with variables of maximal cardiopulmonary exercise testing, such as peak oxygen uptake and oxygen pulse [2, 3], and metabolic equivalent measured at exercise treadmill testing [4]. In addition, it correlates with markers of disease severity in $\mathrm{PAH}$, such as World Health Organization functional class (WHO FC) and pulmonary haemodynamics [3]. Most importantly, the ability of baseline 6MWD to predict mortality has been demonstrated in a 12-week randomised controlled trial of intravenous epoprostenol therapy in idiopathic pulmonary arterial hypertension (IPAH) [5], the only clinical trial showing a survival benefit of $\mathrm{PAH}-$ specific therapies. This was confirmed by subsequent observational studies on the long-term impact of PAH-specific therapies [6-9]. However, there is ongoing debate as to whether \% predicted 6MWD should be used instead of absolute 6MWD as it gives a more accurate an individual.

As with other measurements of physical function, such as lung function and cardiopulmonary exercise capacity, 6MWD varies greatly between individuals due to physiological factors such as age, sex, height and weight, and pathological factors such as cardiopulmonary and musculoskeletal diseases. Adjusting for physiological variation should help clinicians interpret the result of the 6-min walk test (6MWT) and estimate the degree of exercise impairment due to disease in an individual. Several reference equations already exist to calculate \% predicted 6MWD based on an individual's age, sex, height and weight [10-13]. They have been derived from healthy adults sampled from populations in North America and Europe. What is not known is whether \% predicted 6MWD is superior to absolute 6MWD at predicting mortality in $\mathrm{PAH}$. The aim of this study was, therefore, to compare the relative strength of mortality prediction by absolute and \% predicted 6MWD in $\mathrm{PAH}$ reflection of the functional impact of disease on
AFFILIATIONS

Scottish Pulmonary Vascular Unit, Golden Jubilee National Hospital, Clydebank, UK

CORRESPONDENCE

W-T.N. Lee

Scottish Pulmonary Vascular Unit, Level 1

Golden Jubilee National Hospital Beardmore Street

Clydebank

Glasgow G81 4HX UK

E-mail: waiting.lee@nhs.net

Received:

Oct 012009

Accepted after revision: March 162010

First published online:

March 292010 
measured at baseline and on treatment, respectively, using four different published reference equations.

\section{METHODS}

\section{Study population}

The study cohort was derived by a retrospective case note review of patients treated in the Scottish Pulmonary Vascular Unit, which provides the tertiary pulmonary hypertension service for the whole population in Scotland, UK. All consecutive incident cases of IPAH and connective tissue disease-associated pulmonary hypertension (CTDPH) treated with PAH-specific therapy between November 2000 and November 2009 were identified. The diagnosis of PAH was based on right heart catheterisation carried out by the pulmonary vascular team in our institution. $\mathrm{PAH}$ was defined as mean pulmonary artery pressure $\left(\bar{P}_{\mathrm{pa}}\right)>25 \mathrm{mmHg}$ at rest or $>30 \mathrm{mmHg}$ on exercise, with pulmonary capillary wedge pressure $(P \mathrm{pcw}) \leqslant 15 \mathrm{mmHg}$ and pulmonary vascular resistance (PVR) $>3$ Wood units [14]. For patients diagnosed after August 2009, PAH was defined as $\bar{P}_{\text {pa }} \geqslant 25 \mathrm{mmHg}$ at rest with $P$ pcw $\leqslant 15 \mathrm{mmHg}$, and normal or reduced cardiac output [1]. Patients with recorded 6MWD at diagnosis or within 12 months of starting treatment were included in this study. Patients with significant lung disease as defined by forced expiratory volume in $1 \mathrm{~s}<60 \%$ pred or forced vital capacity $<60 \%$ pred or total lung capacity $<60 \%$ pred (based on European Coal and Steel Community reference values) were excluded [15]. Ethical review was considered unnecessary by the West Glasgow research ethics committee.

\section{Treatment and follow-up}

All patients received conventional therapies, such as supplemental oxygen, diuretics and warfarin. PAH-specific monotherapies (prostacyclin analogues, endothelin receptor antagonists and phosphodiesterase- 5 inhibitors) were initiated after confirmation of diagnosis at right heart catheterisation. Treatment response was assessed after a period of 3-6 months by means of clinical assessment, WHO FC and 6MWD. Patients were subsequently followed up at 3-6 month intervals in outpatient clinics. Right heart catheterisation was not routinely repeated during follow-up. Baseline data including age, height, weight, lung function, 6MWD, WHO FC, mean right atrial pressure $\left(\bar{P}_{\text {ra }}\right), \bar{P}_{\text {pa, }}$ PVR, cardiac index $(\mathrm{CI})$ and mixed venous oxygen saturation $\left(\mathrm{SV}, \mathrm{O}_{2}\right)$, and follow-up data including 6MWD, WHO FC and all-cause mortality were collected.

\section{MWT protocol}

$6 \mathrm{MWT}$ was performed by a trained respiratory physiologist on a $20-\mathrm{m}$ corridor with no prior practice walks. Patients were instructed to walk to cover as much ground as possible in 6 min. After 2003, standardised encouragement at 1-min intervals was given as described in the American Thoracic Society guidelines and, before that, 6MWT was performed without encouragement [16]. Predicted 6MWD in each patient was calculated based on their age, sex, height and weight using each of four reference equations (table 1). Absolute 6MWD was expressed as a percentage of the predicted 6MWD.

\section{Statistical analysis}

Statistical analysis was performed using Statview version 5.0.1 (SAS Institute, Cary, NC, USA) and Graphpad Prism version 5.00 (Graphpad Software, La Jolla, CA, USA). Quantitative data were tested for normality using D'Agostino and Pearson omnibus normality test. Parametric data were described by the mean and standard deviation, and compared using paired or unpaired t-test. Nonparametric data were described by the median and interquartile range and compared using MannWhitney U-test. Qualitative data were described by number (\%) and compared by Chi-squared test. The relationship between 6MWD (absolute and each expression of \% predicted 6MWD) and other clinical markers of disease severity was determined by Spearman correlation coefficients. A two-tailed p-value $<0.05$ was defined as significant.

Survival time was defined as time from the date of right heart catheterisation to death or data cut-off on November 30, 2009. Patients who received lung transplantation or who were lost to follow-up were censored at the time of procedure or last clinical contact (clinic visit, telephone contact or renewal of drug prescription). Univariate Cox proportional hazards analysis was carried out to determine the association between all-cause mortality and age, sex, aetiology, lung function, haemodynamics, WHO FC, absolute 6MWD and each expression of $\%$ predicted $6 \mathrm{MWD}$ at baseline, and WHO FC, absolute 6MWD and each expression of \% predicted 6MWD on treatment. Multiple bivariate Cox models, one for absolute $6 \mathrm{MWD}$ and one for each expression of \% predicted $6 \mathrm{MWD}$ at

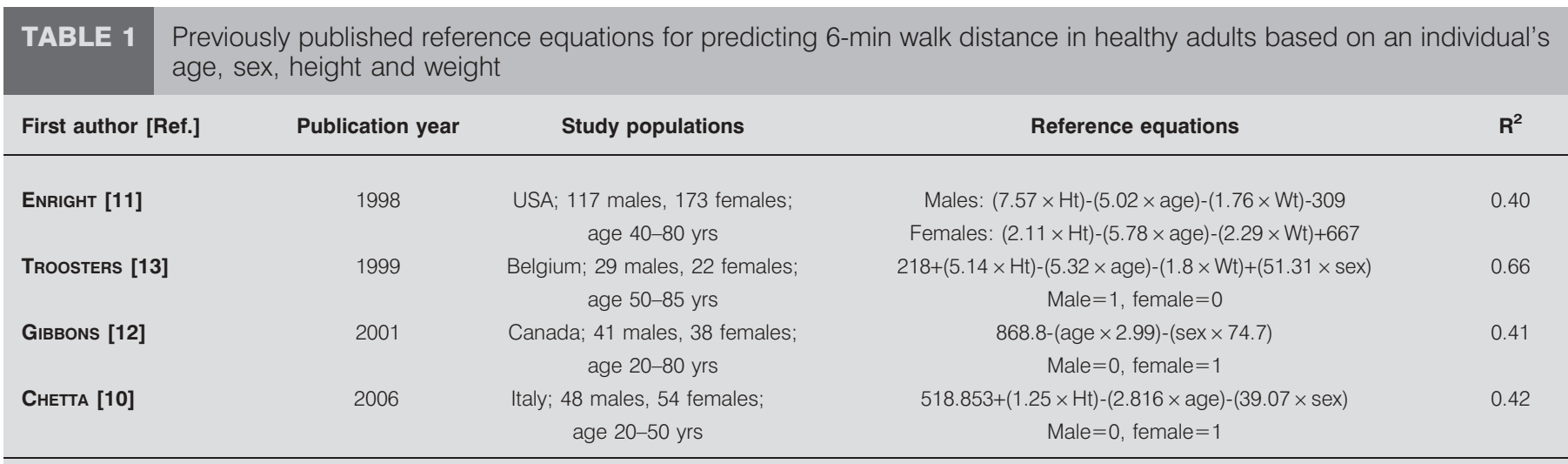

$\mathrm{Ht}$ : height in $\mathrm{cm}$; Wt: weight in $\mathrm{kg} . \mathrm{R}^{2}$ indicates the degree of inter-subject variance explained by the equation. 
baseline, were derived by including variables with $p<0.2$ in the univariate analysis to adjust for the effect of confounding. Correlation between candidate variables and the predictor of primary interest (absolute or \% predicted 6MWD) was checked to limit collinearity. The variables would not be considered for inclusion if $r>0.70$. The same variables were included to adjust $6 \mathrm{MWD}$ in each model to facilitate comparison. The bivariate Cox analysis was repeated for absolute 6MWD and each expression of \% predicted 6MWD on treatment. Date of followup 6MWD was used as the index date for determining survival time for the analysis on treatment.

To compare the relative prognostic strength among expressions of 6MWD against each other at baseline and on treatment respectively, and each corresponding expression of 6MWD at baseline versus on treatment, receiver-operating characteristic

\begin{tabular}{|c|c|c|c|c|}
\hline & \multirow{2}{*}{ All patients } & \multicolumn{2}{|c|}{ Aetiology } & \multirow{2}{*}{ p-value ${ }^{\star}$} \\
\hline TABLE 2 & & IPAH & CTDPH & \\
\hline Patients n & 137 & 86 & 51 & \\
\hline Age $^{\top}$ yrs & $60(22)$ & $60(29)$ & $62(13)$ & 0.18 \\
\hline Females & 95 (69) & $54(63)$ & $41(80)$ & $<0.05$ \\
\hline \multicolumn{5}{|c|}{ Lung function $\%$ pred } \\
\hline FEV1 & 87 (22) & $88(19)$ & $85(24)$ & 0.84 \\
\hline FVC & 99 (23) & $101(22)$ & $98(32)$ & 0.48 \\
\hline$D\llcorner, C O$ & $43(32)$ & $45(38)$ & $41(18)$ & 0.22 \\
\hline \multicolumn{5}{|l|}{ WHO FC } \\
\hline I and II & $22(16)$ & $17(20)$ & $5(10)$ & $<0.05$ \\
\hline III & $104(76)$ & $59(69)$ & $45(88)$ & \\
\hline IV & $11(8)$ & $10(12)$ & 1 (2) & \\
\hline \multicolumn{5}{|l|}{ Haemodynamics } \\
\hline $\bar{P}_{\mathrm{ra}}{ }^{\circ} \mathrm{mmHg}$ & $6(7)$ & $6(7)$ & $7(8)$ & 0.40 \\
\hline $\bar{P} \mathrm{pa}{ }^{\circ} \mathrm{mmHg}$ & $48(19)$ & $50(18)$ & $42(19)$ & $<0.01$ \\
\hline $\mathrm{Cl} l^{\bullet} \cdot \mathrm{min}^{-1} \cdot \mathrm{m}^{-2}$ & $2.1(0.9)$ & $2.2(0.9)$ & $2.0(0.9)$ & 0.92 \\
\hline PVR" Wood units & $10.5(8.4)$ & $10.6(7.8)$ & $9.7(9.9)$ & 0.36 \\
\hline $\mathrm{SV}, \mathrm{O}_{2} \%$ & $63 \pm 8$ & $64 \pm 8$ & $63 \pm 9$ & 0.60 \\
\hline \multicolumn{5}{|l|}{$6 \mathrm{MWD}^{+}$} \\
\hline $\begin{array}{l}\text { Absolute 6MWD m } \\
\text { 6MWD \% pred }\end{array}$ & $264 \pm 111$ & $271 \pm 117$ & $251 \pm 102$ & 0.33 \\
\hline ENRIGHT [11] & $50 \pm 19$ & $50 \pm 20$ & $50 \pm 19$ & 0.95 \\
\hline TROOSTERS [13] & $41 \pm 16$ & $41 \pm 16$ & $42 \pm 15$ & 0.84 \\
\hline GIBBONS [12] & $41 \pm 16$ & $41 \pm 16$ & $40 \pm 15$ & 0.81 \\
\hline CHetTA [10] & $49 \pm 19$ & $49 \pm 20$ & $49 \pm 18$ & 0.92 \\
\hline
\end{tabular}

Data are expressed as mean \pm SD or $n(\%)$, unless otherwise stated; $\bullet$ : median (interquartile range). IPAH: idiopathic pulmonary arterial hypertension; $\mathrm{CTDPH}$ : connective tissue disease-associated pulmonary hypertension; FEV1: forced expiratory volume in $1 \mathrm{~s}$; FVC: forced vital capacity; DL,CO: diffusing capacity of the lung for carbon monoxide; WHO FC: World Health Organization functional class; $\bar{P}_{\text {ra: }}$ mean right atrial pressure; $\bar{P}_{\text {pa: }}$ mean pulmonary artery pressure; $\mathrm{Cl}$ : cardiac index; PVR: pulmonary vascular resistance; $\mathrm{SV}_{1} \mathrm{O}_{2}$ : mixed venous oxygen saturation; 6MWD: 6-min walk distance. *: comparing IPAH versus CTDPH patients; ${ }^{+}: \mathrm{n}=130$.
(ROC) analysis was used to determine their respective area under the ROC curves (AUC) for predicting 2-yr mortality. The significance of differences in AUC was assessed using a paired z-test [17]. Optimal thresholds were identified by selecting the data point closest to the coordinate $x=0$ (1-specificity) and $y=1$ (sensitivity) on the ROC curves.

TABLE 3 Univariate Cox proportional hazards analysis

\begin{tabular}{|c|c|c|}
\hline & HR $(95 \%$ Cl) & p-value \\
\hline Age at diagnosis (per decade increase) & $1.43(1.11-1.86)$ & $<0.005$ \\
\hline \multicolumn{3}{|l|}{ Sex } \\
\hline Female & $0.61(0.32-1.19)$ & 0.14 \\
\hline \multicolumn{3}{|l|}{ Male (reference) } \\
\hline \multicolumn{3}{|l|}{ Lung function at baseline } \\
\hline FEV1 (per 10\% pred increase) & $1.09(0.91-1.31)$ & 0.36 \\
\hline FVC (per 10\% pred increase) & $1.07(0.91-1.26)$ & 0.42 \\
\hline DL,CO (per 10\% pred increase) & $0.75(0.63-0.90)$ & $<0.005$ \\
\hline \multicolumn{3}{|l|}{ WHO FC at baseline } \\
\hline I/II & $0.60(0.18-2.02)$ & 0.41 \\
\hline III & $0.63(0.24-1.67)$ & 0.34 \\
\hline \multicolumn{3}{|l|}{ IV (reference) } \\
\hline \multicolumn{3}{|l|}{ WHO FC on treatment } \\
\hline I/II & $0.13(0.04-0.43)$ & $<0.001$ \\
\hline III & $0.28(0.09-0.89)$ & $<0.05$ \\
\hline \multicolumn{3}{|l|}{ IV (reference) } \\
\hline \multicolumn{3}{|l|}{ Aetiology } \\
\hline CTDPH & $1.63(0.87-3.06)$ & 0.12 \\
\hline \multicolumn{3}{|l|}{ IPAH (reference) } \\
\hline \multicolumn{3}{|l|}{ Haemodynamics at baseline } \\
\hline $\bar{P}_{\text {ra }}$ (per 5 mmHg increase) & $1.19(0.90-1.58)$ & 0.21 \\
\hline $\bar{P}$ pa (per 5 mmHg increase) & $1.01(0.91-1.12)$ & 0.81 \\
\hline $\mathrm{Cl}$ (per $1 \mathrm{~L} \cdot \mathrm{min}^{-1} \cdot \mathrm{m}^{-2}$ increase) & $0.83(0.49-1.39)$ & 0.47 \\
\hline PVR (per 1 Wood unit increase) & $1.01(0.96-1.06)$ & 0.68 \\
\hline $\mathrm{SV}, \mathrm{O}_{2}$ (per $5 \%$ increase) & $0.93(0.73-1.19)$ & 0.56 \\
\hline \multicolumn{3}{|l|}{$6 \mathrm{MWD}$ at baseline ${ }^{\#}$} \\
\hline Absolute 6MWD (per 50 m increase) & $0.78(0.67-0.95)$ & $<0.005$ \\
\hline \multicolumn{3}{|l|}{$6 \mathrm{MWD} \%$ pred (per 10\% increase) } \\
\hline ENRIGHT [11] & $0.83(0.69-0.99)$ & $<0.05$ \\
\hline TROOSTERS [13] & $0.77(0.62-0.96)$ & $<0.05$ \\
\hline GiBBONS [12] & $0.74(0.61-0.90)$ & $<0.005$ \\
\hline СHETTA [10] & $0.79(0.66-0.94)$ & $<0.01$ \\
\hline \multicolumn{3}{|l|}{ 6MWD on treatment } \\
\hline Absolute 6MWD (per 50 m increase) & $0.74(0.64-0.90)$ & $<0.005$ \\
\hline \multicolumn{3}{|l|}{$6 \mathrm{MWD} \%$ pred (per 10\% increase) } \\
\hline ENRIGHT [11] & $0.75(0.61-0.93)$ & $<0.01$ \\
\hline TROOSTERS [13] & $0.68(0.53-0.89)$ & $<0.005$ \\
\hline GiBBONS [12] & $0.67(0.53-0.85)$ & $<0.005$ \\
\hline CHETTA [10] & $0.72(0.59-0.88)$ & $<0.005$ \\
\hline
\end{tabular}

HR: hazard ratio; FEV1: forced expiratory volume in $1 \mathrm{~s}$; FVC: forced vital capacity; $D \mathrm{~L}, \mathrm{CO}$ : diffusing capacity of the lung for carbon monoxide; WHO FC: World Health Organization functional class; CTDPH: connective tissue diseaseassociated pulmonary hypertension; IPAH: idiopathic pulmonary arterial hypertension; $\bar{P}_{\mathrm{ra}}$ : mean right atrial pressure; $\bar{P}_{\mathrm{pa}}$ : mean pulmonary artery pressure; $\mathrm{Cl}$ : cardiac index; PVR: pulmonary vascular resistance; $\mathrm{SV}, \mathrm{O}_{2}$; mixed venous saturation; 6MWD: 6-min walk distance. ${ }^{\#}: n=130 ;{ }^{\circ}: n=110$. 
a) Absolute 6MWD

Unadjusted
Variable adjusted for
$D \mathrm{~L}, \mathrm{co}$ at baseline
$\bar{P}_{\text {ra at baseline }}$
Sex
Aetiology
Age

$\begin{array}{lllllllll}0.5 & 0.6 & 0.7 & 0.8 & 0.9 & 1.0 & 1.1 & 1.2\end{array}$

$\mathrm{HR}$ (per $50 \mathrm{~m}$ increase)

c) TROOSTERS et al. [13] \% predicted 6MWD

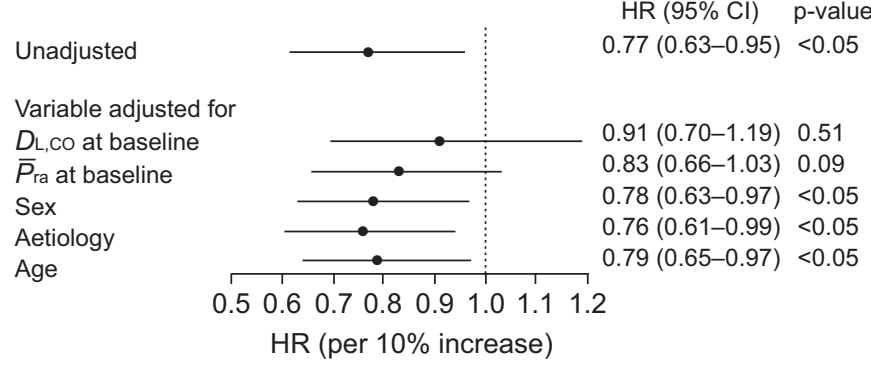

e) CHETTA et al. [10] \% predicted 6MWD

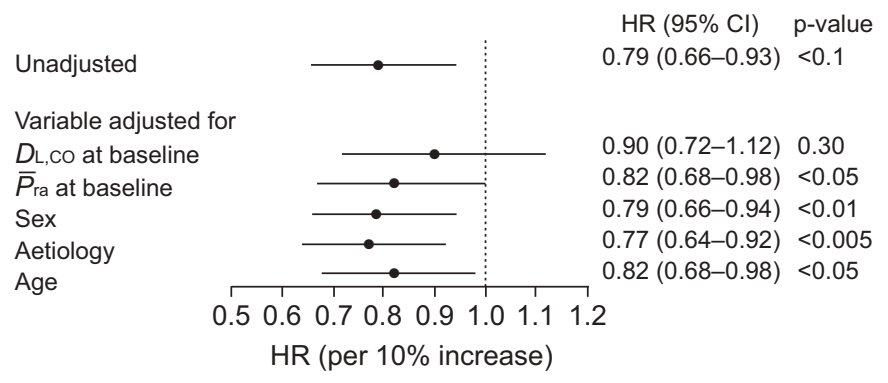

RESULTS

\section{Patient characteristics}

137 patients were included in the cohort (IPAH 86, CTDPH 51). The baseline characteristics of the study patients are outlined in table 2. During follow-up (median 2 yrs; range 1.2 months to $8.8 \mathrm{yrs}$ ), 41 patients died from all causes (IPAH 20, CTDPH 21), two patients were lost to follow-up and two patients received lung transplantation. After receiving PAH-specific monotherapy $(7 \%$ prostacyclin analogues, $46 \%$ endothelin receptor antagonists and $47 \%$ phosphodiesterase- 5 inhibitors) for $4.1 \pm 1.8$ months (median 3.5 months; range 2.211 months), absolute 6MWD and each expression of \% predicted 6MWD were $311 \pm 111 \mathrm{~m}, 59 \pm 18 \%$ (ENRIGHT and SHERrILl [11]), $48 \pm 15 \%$ (TROOSTERS et al. [13]), $48 \pm 16 \%$ (GIBBONS et al. [12]) and $58 \pm 19 \%$ (CHETTA et al. [10]) respectively ( $\mathrm{n}=110$, all $\mathrm{p}<0.0001$ versus baseline). The distribution of absolute 6MWD at baseline, on treatment and the distance changed are shown in figure 1 in the online supplementary material. On treatment, $41 \%$ of patients were in WHO FC II, $54 \%$ in III and $4 \%$ in IV $(n=123 ; p<0.005$ versus baseline). At the time of death or censoring, $69 \%$ of patients remained on monotherapy and $31 \%$ were on combination therapy. b) ENRIGHT and SHERRILL [11] \% predicted 6MWD

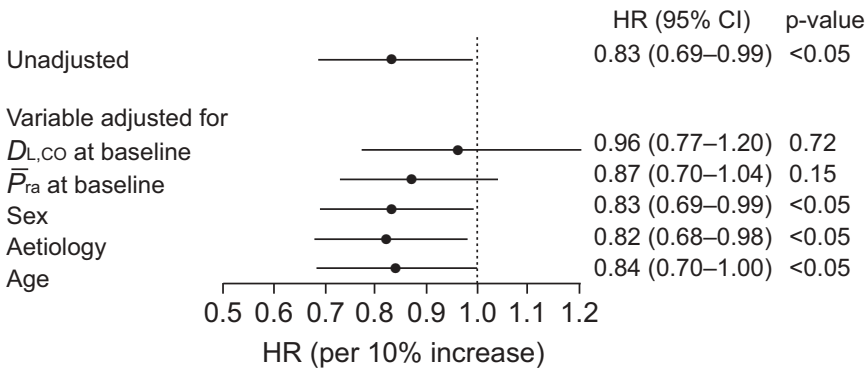

d) GiBBons et al. [12] \% predicted 6MWD

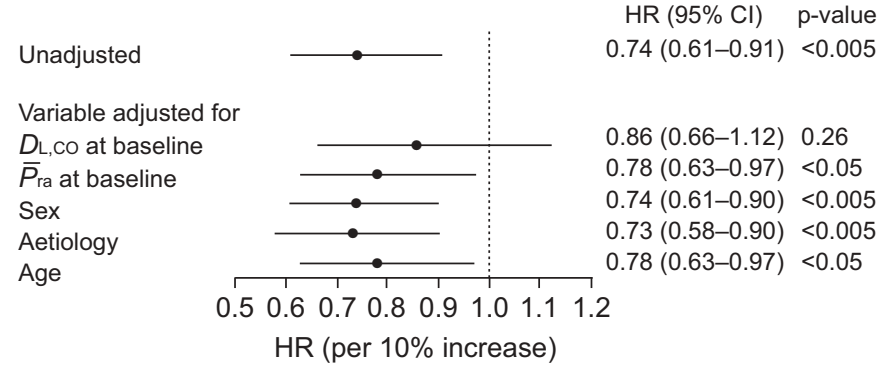

FIGURE 1. Bivariate Cox proportional hazards models of absolute and $\%$ predicted 6-min walk distance (6MWD) by four different reference equations at baseline. $D \mathrm{~L}, \mathrm{CO}$ : diffusing capacity of the lung for carbon monoxide; $\bar{P}$ ra: mean right atrial pressure. The unadjusted and adjusted hazard ratios (HRs) of 6MWD are indicated by closed circles and the $95 \%$ confidence interval by error bars. Adjusted HRs of $D$ L,CO are $0.76-0.79$ per $10 \%$ predicted increase; all $p<0.05$.

\section{Correlation with other markers of disease severity at baseline}

Baseline absolute and \% predicted 6MWD correlated weakly with $\bar{P}_{\text {ra }}$ (absolute 6MWD $\mathrm{r}=-0.23 ; \mathrm{p}<0.01$ versus \% predicted $6 \mathrm{MWD}$ by all equations $\mathrm{r}=-0.23$ to $-0.25 ; \mathrm{p}<0.01), \mathrm{CI}(\mathrm{r}=0.24$; $\mathrm{p}<0.01$ versus $\mathrm{r}=0.21$ to $0.22 ; \mathrm{p}<0.05), S \mathrm{~V}, \mathrm{O}_{2}(\mathrm{r}=0.21 ; \mathrm{p}<0.05$ versus $\mathrm{r}=0.19$ to $0.21 ; \mathrm{p}<0.05)$ and WHO FC $(\mathrm{r}=-0.33$; $\mathrm{p}<0.0005$ versus $\mathrm{r}=-0.32$ to $-0.34 ; \mathrm{p}<0.005)$. Only $6 \mathrm{MWD} \%$ predicted by ENRIGHT and SHERRILL [11] and TROOSTERS et al. [13] equations correlated weakly with PVR $(r=-0.23$ and $\mathrm{r}=-0.19$; both $\mathrm{p}<0.05)$. Neither absolute 6MWD nor \% predicted 6MWD correlated with $\bar{P}_{\text {pa. }}$.

\section{Independent effect on mortality}

Age and diffusing capacity of the lung for carbon monoxide $(D \mathrm{~L}, \mathrm{CO})$ at baseline, absolute and \% predicted 6MWD at baseline and on treatment, and WHO FC on treatment predicted all-cause mortality in univariate Cox proportional hazards analysis (table 3). The predictive value of absolute and $\%$ predicted $6 \mathrm{MWD}$ at baseline remained significant after adjusting for age, sex and aetiology of PAH but not DL,CO in bivariate Cox proportional hazards analysis (fig. 1). Absolute 6MWD, and 6MWD \% predicted by GiBBONs et al. [12] and 


\section{a) Absolute 6MWD}

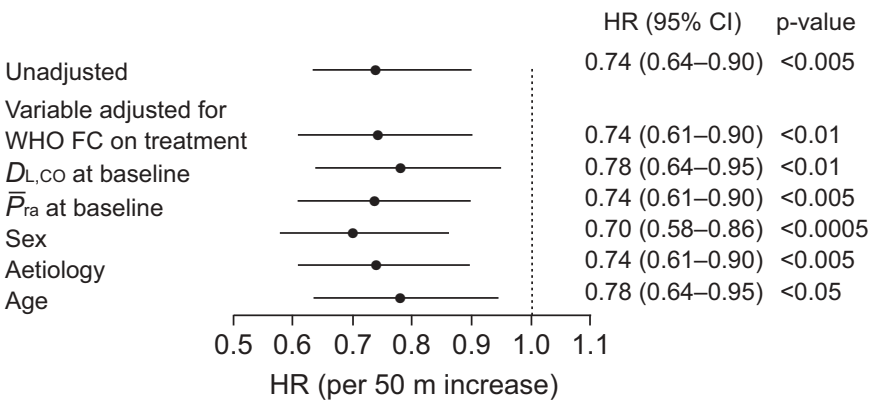

c) TROOsters et al. [13] \% predicted 6MWD

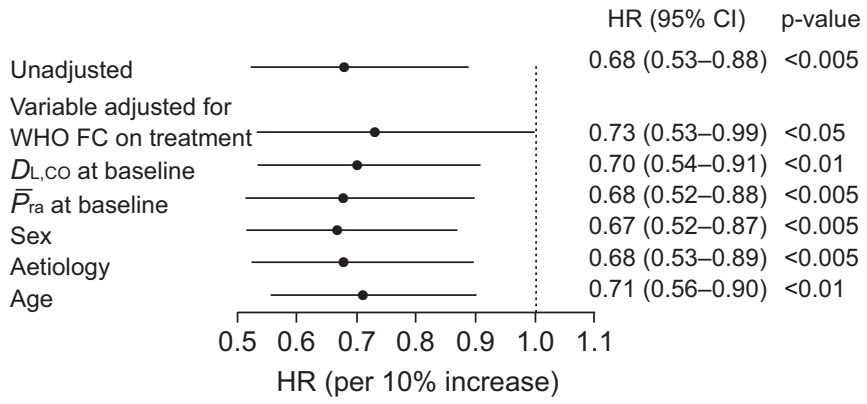

e) CHETTA et al. [10] \% predicted 6MWD

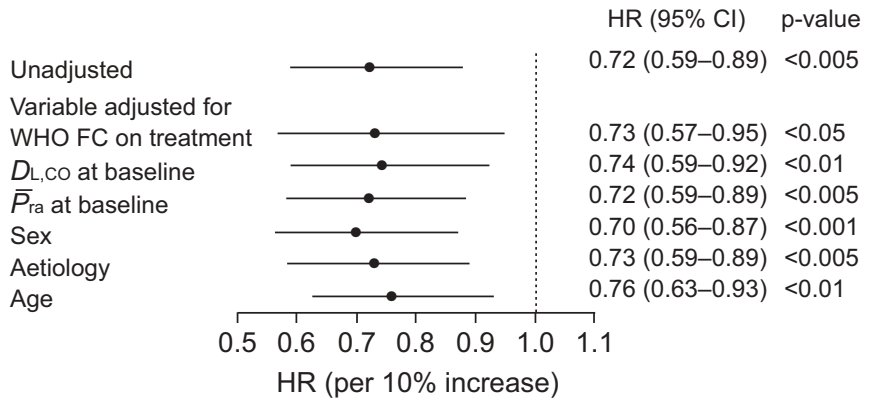

CHETTA et al. [10] predicted mortality after adjusting for $\bar{P}_{\text {ra but }}$ 6MWD \% predicted by ENRIGHT and SHERRILL [11] or TROOSTERS et al. [13] did not. On treatment, absolute and \% predicted 6MWD was predictive of mortality independently of WHO FC (except ENRIGHT and SHERRILL [11] equation), age, sex, aetiology, baseline $D \mathrm{~L}, \mathrm{CO}$ and $\bar{P}_{\text {ra }}$ (fig. 2). When combined with absolute or \% predicted 6MWD on treatment, DL,CO at baseline no longer predicted mortality.

\section{Relative strength of mortality prediction}

ROC curves of absolute 6MWD and \% predicted 6MWD by each of four reference equations at baseline and on treatment are shown in figure 3 . There were no significant differences in AUC of absolute versus each expression of \% predicted 6MWD for predicting 2-yr mortality at baseline or on treatment (table 4). In addition, there were no significant differences in AUC among \% predicted 6MWD by the four reference equations. The optimal thresholds of absolute 6MWD and each expression of \% predicted 6MWD at baseline and on treatment for predicting 2-yr mortality had modest sensitivity and specificity. The thresholds of absolute 6MWD and 6MWD b) ENRIGHT and SHERRILL [11] \% predicted 6MWD

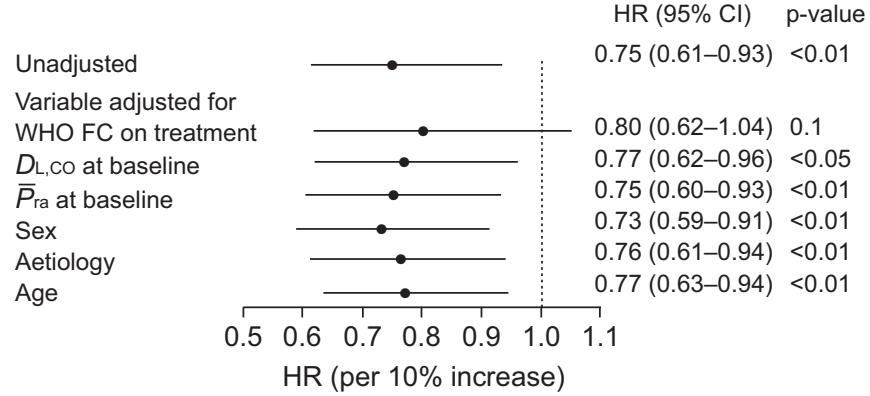

d) GiBBONs et al. [12] \% predicted 6MWD

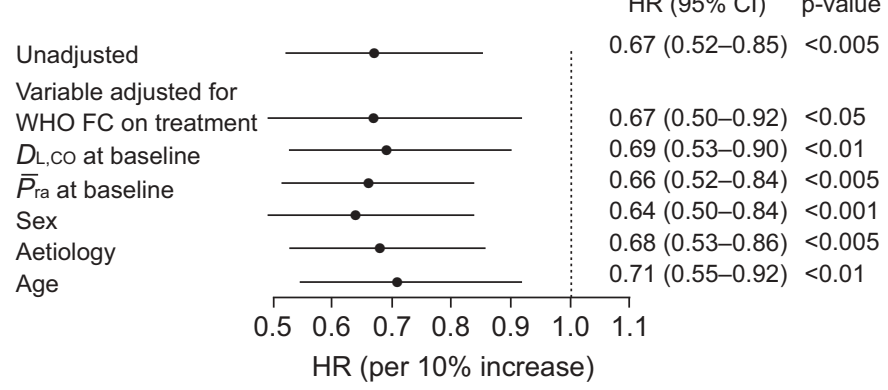

FIGURE 2. Bivariate Cox proportional hazards models of absolute and \% predicted 6-min walk distance (6MWD) by four different reference equations on treatment. WHO FC: World Health Organization functional class; DL,Co: diffusing capacity of the lung for carbon monoxide; $\bar{P}_{\text {ra: }}$ mean right atrial pressure. The unadjusted and adjusted hazard ratios (HRs) of 6MWD are indicated by closed circles and the $95 \%$ confidence interval by error bars.

$\%$ predicted by equations from TrOOSTERs et al. [13], GiBBONS et al. [12] and CHETTA et al. [10] on treatment had slightly higher sensitivity and specificity than those at baseline, but the respective differences in AUC between baseline and treatment did not reach statistical significance. This analysis was repeated including only patients diagnosed from 2003 onwards as the $6 \mathrm{MWT}$ protocol changed at that point and the results were similar (table 1 and fig. 2 in the online supplementary material).

\section{DISCUSSION}

This study addresses an important clinical issue regarding the role of $\%$ predicted $6 \mathrm{MWD}$ in the management of PAH. The data analysis showed that \% predicted 6MWD at baseline derived from currently available reference equations predicts all-cause mortality but its prognostic ability is not superior to that of absolute 6MWD despite adjusting for physiological inter-subject variance. In addition, there is no difference in the prognostic ability between each of the four studied reference equations. Assuming that the long-term prognosis of patients improves with PAH-specific therapy, 6MWD on treatment 

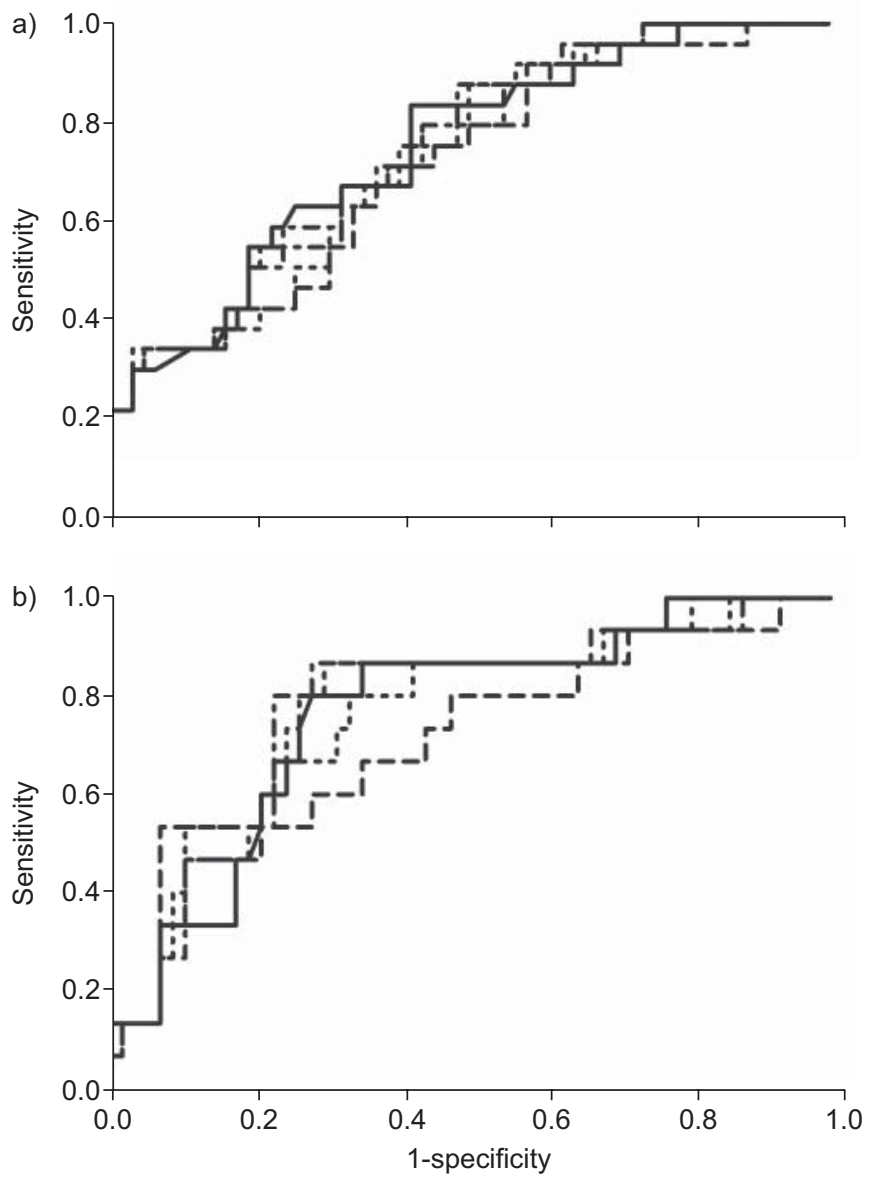

FIGURE 3. Receiver-operating characteristic curves of absolute and \% predicted 6-min walk distance (6MWD) by four different reference equations in predicting 2-yr mortality a) at baseline and b) on treatment. ——: absolute 6MWD; - - - -: ENRIGHT and SHERRILL [11] \% predicted 6MWD; - - - - -: TROOSTERS et al. [13] \% predicted 6MWD; - - - - -: GIBBONs et al. [12] \% predicted 6MWD; - - - - -: CHETTA et al. [10] \% predicted 6MWD. All p>0.05 when area under the receiver-operating characteristic curves is compared among expressions of 6MWD at baseline and on treatment, respectively, by a paired z-test

may be a stronger predictor of mortality than that at baseline, and hence the difference in the relative prognostic strength between absolute and \% predicted 6MWD may be more apparent on treatment. However, this is not supported by the results of this analysis. \% predicted 6MWD was not more predictive of mortality than absolute 6MWD on treatment.

$\%$ predicted 6MWD calculated from the equations of TROOSTERs et al. [13] and GiBBONS et al. [12] was slightly lower than that from ENRIGHT and SHERRILL [11] and CHETTA et al. [10] equations. This disparity is due to differences in the $6 \mathrm{MWT}$ protocol used in the studies, such as the length of corridor, type and frequency of encouragement and the number of practice walks. A 50-m corridor was used by TROOSTERS et al. [13] and the best of four walks by GiBBONs et al. [12], which resulted in higher predicted absolute 6MWD. The equations were also derived from healthy adults in different age groups. Patients younger than 40 yrs of age were included by CHETTA et al. [10] and GibBONs et al. [12] only. This highlights the importance of giving consideration to the characteristics of the study population and the $6 \mathrm{MWT}$ protocol used in deriving the reference equation when applying predicted values. The American Thoracic Society guidelines on 6MWT recognise that $\%$ predicted $6 \mathrm{MWD}$ is under-utilised due to a lack of optimal reference equations [16]. Only 40-66\% of physiological inter-subject variance of $6 \mathrm{MWD}$ is explained by the currently available equations. Therefore, there are other factors influencing the performance at the 6MWT that have not been identified or accounted for. This may partly explain the failure to demonstrate the superiority of \% predicted 6MWD by any of the four reference equations to absolute 6MWD in predicting mortality.

There are other potential explanations for the negative findings in this study. It might be possible for patients with low or high $6 \mathrm{MWD}$ to dominate the analysis, thereby obscuring any prognostic difference between absolute and \% predicted $6 \mathrm{MWD}$. However, this was not the case as the 6MWD data were normally distributed with few patients at either end of the range. The use of all-cause mortality rather than diseasespecific mortality may also be relevant as this could shift the comparison of prognostic strength in favour of absolute $6 \mathrm{MWD}$. For example, consider the case of a 40-yr-old male with severe PAH (height $186 \mathrm{~cm}$, weight $90 \mathrm{~kg}$ ) and an 80-yrold female with mild PAH (height $157 \mathrm{~cm}$, weight $60 \mathrm{~kg}$ ) both of whom walk the same 6MWD of $300 \mathrm{~m}$ and survive for $5 \mathrm{yrs}$. This walk distance corresponds to $40 \%$ and $75 \%$ pred, respectively, using the ENRIGHT and SHERRILL [11] equation, reflecting more severe disease in the male. The 40-yr-old male is more likely to die from PAH, whereas the 80 -yr-old female is more likely to die from other causes, as she approaches the natural limit of life expectancy. In this scenario, absolute $6 \mathrm{MWD}$ is the better predictor of all-cause mortality as it has automatically incorporated the influence of age on mortality, with age being the most important physiological determinant of 6MWD. Conversely, \% predicted 6MWD would be expected to perform better at predicting disease-specific mortality. Allcause mortality was used in this study to avoid the subjectivity associated with disease-specific mortality.

The fact that there is a considerable amount of unexplained physiological variance in 6MWD raises concerns about its reliability as an outcome measure in PAH. Some clinicians propose that 6MWD should be used instead to set a treatment goal in individual patients based on their starting parameters, where inter-subject variance is not as relevant. To set an appropriate goal, the knowledge of predicted 6MWD in an individual is essential. Therefore, despite having no added prognostic information, \% predicted 6MWD would help clinicians to ascertain the degree of improvement achievable should a goal-oriented treatment strategy be adopted. There is also a suggestion that $6 \mathrm{MWD}$ should be replaced altogether by alternative outcome measures that are more directly linked to right heart function or pulmonary haemodynamics, such as cardiac magnetic resonance or noninvasive cardiac output measurements. There is no doubt that more robust exercise outcome measures than 6MWD are needed in PAH, but until they are developed and validated, 6MWD on balance remains the best available option in the appropriate setting given its proven prognostic value and simplicity. 


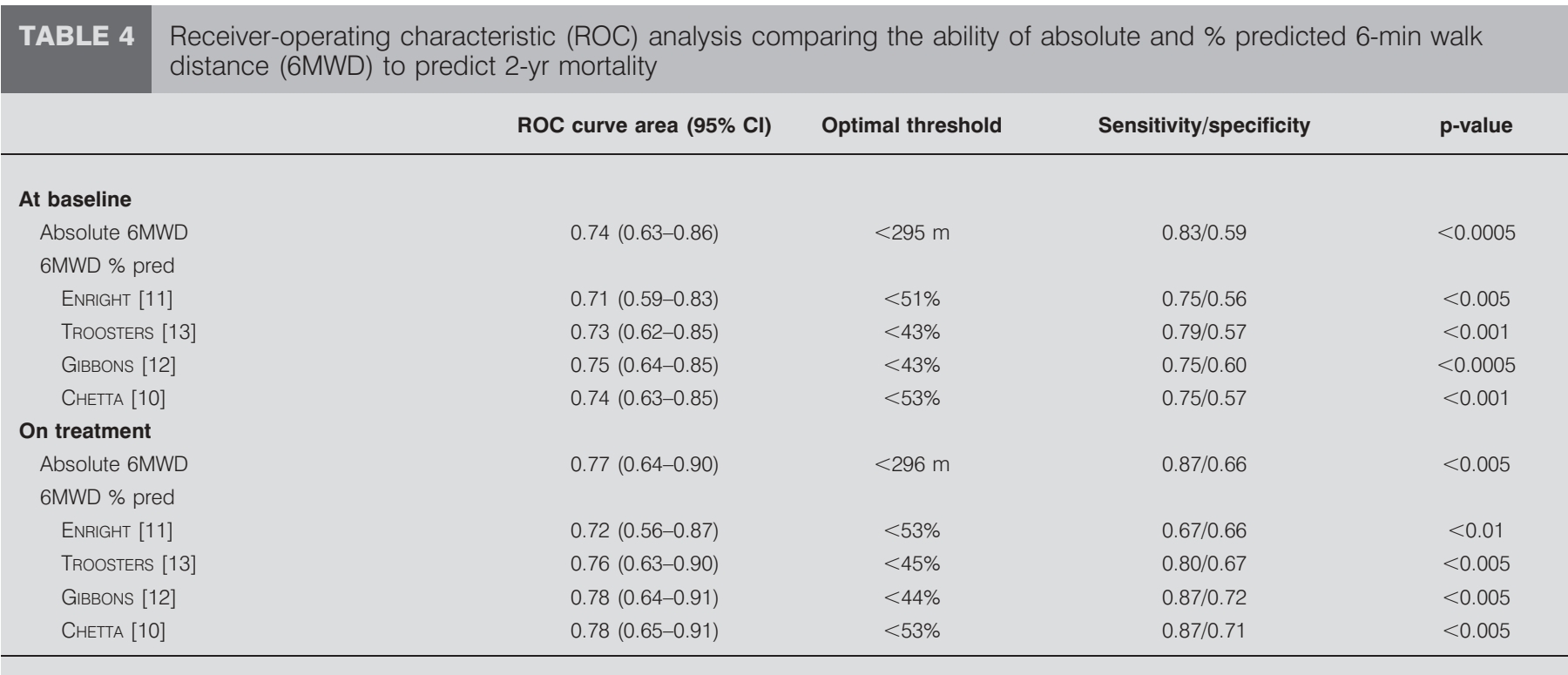

The prognostic value of $\mathrm{DL}, \mathrm{CO}$ in $\mathrm{PAH}$ has been explored in a large retrospective study that showed that $\mathrm{DL}, \mathrm{CO}$ at baseline predicted mortality after adjusting for age, CTDPH aetiology, WHO FC, haemodynamics and lung abnormalities on computed tomography [18]. However, the finding of $D \mathrm{~L}, \mathrm{CO}$ predicting mortality more strongly than 6MWD at baseline in this analysis is of interest and to our knowledge has not been reported before. It would be interesting to explore the predictive value of $\mathrm{DL}, \mathrm{CO}$ on treatment but such data were not available in this study cohort.

The study has a number of limitations. First, selection bias cannot be excluded as this is a retrospective study and gaps due to missing data are unavoidable. $14(10 \%)$ patients with no $6 \mathrm{MWD}$ data at baseline or within 12 months of starting treatment were excluded from the analysis. This is unlikely to have affected the main outcome of the analysis, namely the comparison between absolute and \% predicted 6MWD. Secondly, patients were recruited over a relatively long period of time during which treatment approaches had evolved. This may have led to differences in prognosis of patients diagnosed in the earlier years compared to those diagnosed more recently. While this cannot be excluded, it is worth pointing out that there is as yet no conclusive evidence to support improved prognosis with the widespread use of oral agents in the past few years compared to the epoprostenol era. Thirdly, the $6 \mathrm{MWT}$ protocol changed during the study period and encouragement may have significantly influenced 6MWD. However, the results were similar with patients diagnosed after 2003 only. Finally, the reference equations were derived from healthy populations with differing age groups and hence no single equation was perfectly applicable to the study patients.

In conclusion, \% predicted 6MWD may help clinicians quantify the functional impact of disease on an individual, but its prognostic ability is not superior to that of absolute 6MWD. In addition, the prognostic ability of currently available reference equations is similar. To explore the role of $\%$ predicted 6MWD in the management of PAH further, efforts should be made to develop more robust reference equations using a standardised 6MWT protocol and sampling healthy adults from more representative populations, particularly in relation to age.

\section{STATEMENT OF INTEREST}

A statement of interest for this study can be found at www.erj ersjournals.com/site/misc/statements.xhtml

\section{REFERENCES}

1 Galie N, Hoeper MM, Humbert M, et al. Guidelines for the diagnosis and treatment of pulmonary hypertension: The Task Force for the Diagnosis and Treatment of Pulmonary Hypertension of the European Society of Cardiology (ESC) and the European Respiratory Society (ERS), endorsed by the International Society of Heart and Lung Transplantation (ISHLT). Eur Heart J 2009; 30: 2493-2537.

2 Oudiz RJ, Barst RJ, Hansen JE, et al. Cardiopulmonary exercise testing and six-minute walk correlations in pulmonary arterial hypertension. Am J Cardiol 2006; 97: 123-126.

3 Miyamoto S, Nagaya N, Satoh T, et al. Clinical correlates and prognostic significance of six-minute walk test in patients with primary pulmonary hypertension. Comparison with cardiopulmonary exercise testing. Am J Respir Crit Care Med 2000; 161: $487-492$.

4 Gomberg-Maitland M, Huo D, Benza RL, et al. Creation of a model comparing 6-minute walk test to metabolic equivalent in evaluating treatment effects in pulmonary arterial hypertension. J Heart Lung Transplant 2007; 26: 732-738.

5 Barst RJ, Rubin LJ, Long WA, et al. A comparison of continuous intravenous epoprostenol (prostacyclin) with conventional therapy for primary pulmonary hypertension. The Primary Pulmonary Hypertension Study Group. N Engl J Med 1996; 334: 296-302.

6 Hoeper MM, Gall H, Seyfarth HJ, et al. Long-term outcome with intravenous iloprost in pulmonary arterial hypertension. Eur Respir J 2009; 34: 132-137.

7 McLaughlin VV, Sitbon O, Badesch DB, et al. Survival with firstline bosentan in patients with primary pulmonary hypertension. Eur Respir J 2005; 25: 244-249. 
8 Provencher S, Sitbon O, Humbert M, et al. Long-term outcome with first-line bosentan therapy in idiopathic pulmonary arterial hypertension. Eur Heart J 2006; 27: 589-595.

9 Sitbon $\mathrm{O}$, Humbert $\mathrm{M}$, Nunes $\mathrm{H}$, et al. Long-term intravenous epoprostenol infusion in primary pulmonary hypertension: prognostic factors and survival. J Am Coll Cardiol 2002; 40: 780-788.

10 Chetta A, Zanini A, Pisi G, et al. Reference values for the 6-min walk test in healthy subjects 20-50 years old. Respir Med 2006; 100: 1573-1578.

11 Enright PL, Sherrill DL. Reference equations for the six-minute walk in healthy adults. Am J Respir Crit Care Med 1998; 158: 1384-1387.

12 Gibbons WJ, Fruchter N, Sloan S, et al. Reference values for a multiple repetition 6-minute walk test in healthy adults older than 20 years. J Cardiopulm Rehabil 2001; 21: 87-93.

13 Troosters T, Gosselink R, Decramer M. Six minute walking distance in healthy elderly subjects. Eur Respir J 1999; 14: 270-274.
14 Galie N, Torbicki A, Barst R, et al. Guidelines on diagnosis and treatment of pulmonary arterial hypertension. The Task Force on Diagnosis and Treatment of Pulmonary Arterial Hypertension of the European Society of Cardiology. Eur Heart J 2004; 25: 2243-2278.

15 Quanjer PH, Tammeling GJ, Cotes JE, et al. [Lung volumes and forced ventilatory flows. Work Group on Standardization of Respiratory Function Tests. European Community for Coal and Steel. Official position of the European Respiratory Society]. Rev Mal Respir 1994; 11: Suppl. 3, 5-40.

16 Brooks D, Solway S, Gibbons WJ. ATS statement on six-minute walk test. Am J Respir Crit Care Med 2003; 167: 1287.

17 Hanley JA, McNeil BJ. A method of comparing the areas under receiver operating characteristic curves derived from the same cases. Radiology 1983; 148: 839-843.

18 Chandra S, Shah SJ, Thenappan T, et al. Carbon monoxide diffusing capacity and mortality in pulmonary arterial hypertension. J Heart Lung Transplant 2010; 29: 181-187. 